\title{
Bis(oxazolines) based on glycopyranosides - steric, configurational and conformational influences on stereoselectivity
}

\author{
Tobias Minuth and Mike M. K. Boysen*
}

Open Access

\author{
Full Research Paper \\ Address: \\ Institute of Organic Chemistry, Gottfried-Wilhelm-Leibniz University of \\ Hannover, Schneiderberg 1B, D-30167 Hannover, Germany \\ Email: \\ Mike M. K. Boysen* - mike.boysen@oci.uni-hannover.de \\ * Corresponding author \\ Keywords: \\ asymmetric synthesis; carbohydrates; copper; cyclopropanation; \\ ligand design
}

\author{
Beilstein Journal of Organic Chemistry 2010, 6, No. 23 \\ doi:10.3762/bjoc.6.23 \\ Received: 08 December 2009 \\ Accepted: 18 February 2010 \\ Published: 04 March 2010 \\ Guest Editor: T. K. Lindhorst \\ (C) 2010 Minuth and Boysen; licensee Beilstein-Institut. \\ License and terms: see end of document.
}

\begin{abstract}
In previous studies we found that the asymmetric induction of bis(oxazolines) based on D-glucosamine strongly depended on the steric demand of the 3-O-substituents. To further probe the impact of the 3-position of the pyranose scaffold, we prepared 3-epimerised and 3-defunctionalised versions of these ligands as well as a 3-O-formyl derivative. Application of these new ligands in asymmetric cyclopropanation revealed strong steric and configurational effects of position 3 on asymmetric induction, further dramatic effects of the pyranose conformation were also observed.
\end{abstract}

\section{Introduction}

The design and optimisation of chiral ligands for metal catalysed transformations is of crucial importance for stereoselective synthesis and is therefore an active field of research. In this context, carbohydrates are interesting, even if comparatively rarely used as starting materials for the preparation of new chiral ligand structures. Today, 30 years after the first reports on carbohydrate-based ligands [1-4], the potential of saccharide compounds in this area is more and more appreciated [5-12].

Chiral bis(oxazolines) (Box) are very efficient ligands for many asymmetric transformations $[13,14]$. Even though N-acylated derivatives of D-glucosamine easily form bicyclic carbohydrate oxazolines, until recently only a few examples of mono(oxazoline) ligands [15-17] and the corresponding bis(oxazolines) [18] based on this monosaccharide have appeared in the literature. In the course of our work we have introduced new glucosaminederived bis(oxazolines) 2a-c with uniform protective groups on all oxygen functions [19-21] and derivatives 3a-f with cyclic 4,6-O-benzylidene protection as well as various other 3-Osubstituents that differ in steric demand and electronic nature [20,21].

These ligands were subsequently employed in the asymmetric cyclopropanation [22,23] of styrene (4) with ethyl diazoacetate (5). Our results revealed a strong dependence of 
the enantioselectivity on both the steric bulk and electronic nature of the $O$-substituents in ligands $\mathbf{2 a}-\mathbf{c}$ and $\mathbf{3 a}-\mathbf{f}$. Furthermore, the conformation of the pyranose scaffold - a twist conformation for ligands $2 \mathbf{a}-\mathbf{c}$ without 4,6-O-benzylidene protection (Scheme 1, conformer A) and a partially chair-like con- formation for ligands 3a-f (Scheme 1, conformer $\mathbf{B}$ ) fixed by the annulated 4,6-O-benzylidene group - has a direct impact on the enantioselectivity of the reaction. For ligands $\mathbf{3 a}-\mathbf{f}$ with cyclic protection, a decrease in the bulk of the 3-O-residues led to an improvement in stereoselectivity, while the opposite trend

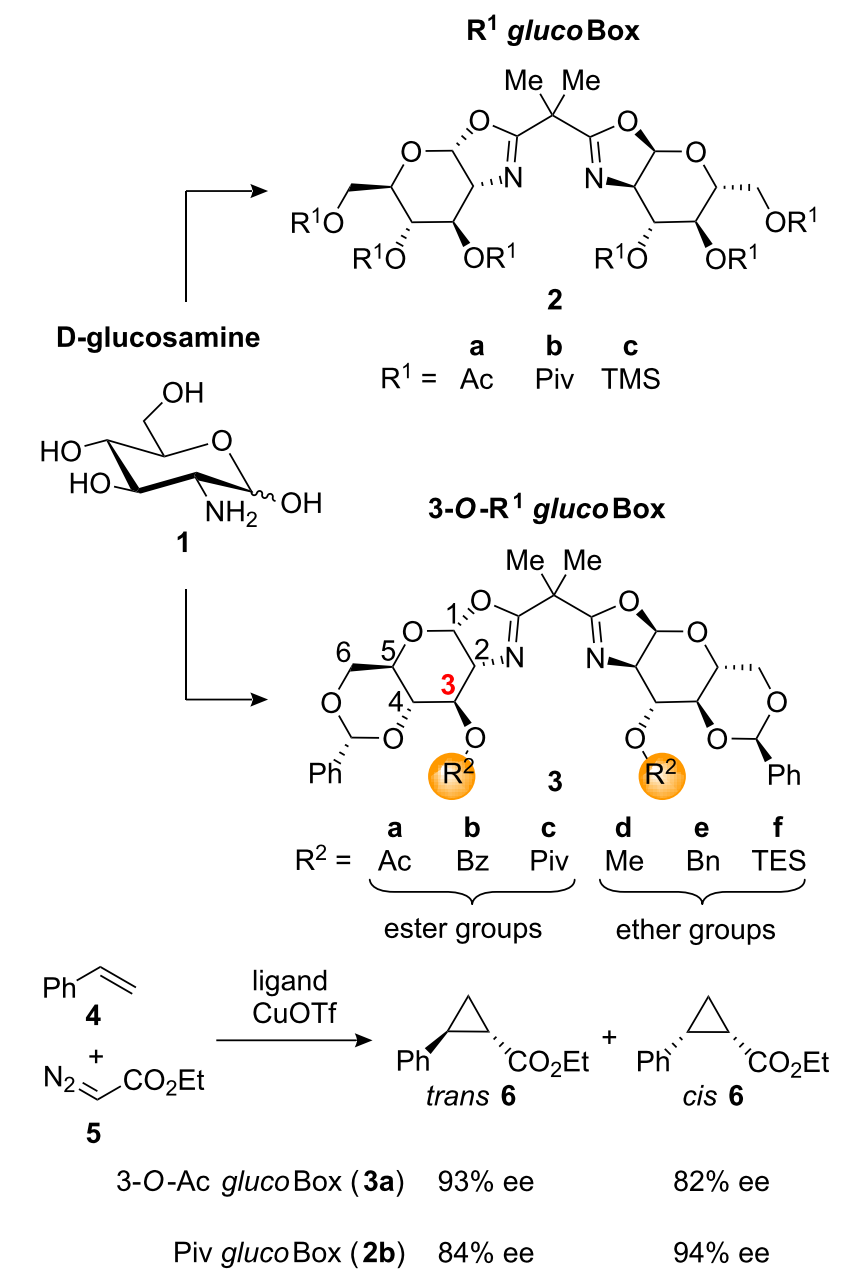

Scheme 1: New glucosamine-based bis(oxazoline) ligands with their pyranose conformation and application in asymmetric cyclopropanation.

(I) Inversion of the stereocentre at position 3

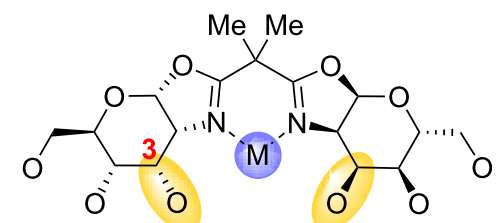

allo-configured bis(oxazolines)
(II) Removal of the stereocentre at position 3

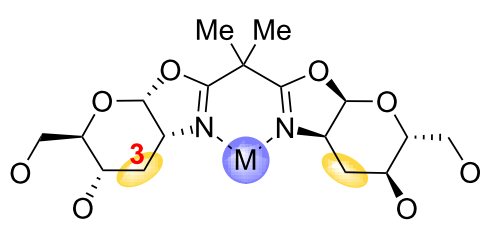

3-deoxygenated bis(oxazolines)

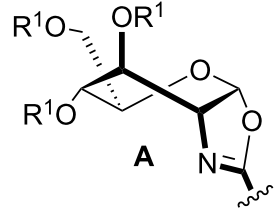

twist conformation of pyranose scaffold without 4,6-O-benzylidene acetal

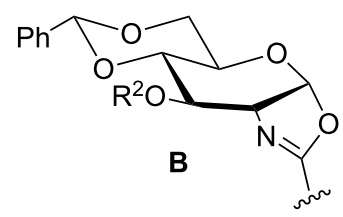

pyranose scaffold partially fixed in chair-like conformation by $4,6-0$ benzylidene acetal

Figure 1: Planned modifications at pyranose position 3 of carbohydrate bis(oxazolines). 
was observed for counterparts $\mathbf{2 a - c}$ with acyclic 4,6-O-protection. Moreover, ester modified ligands $\mathbf{2 a}, \mathbf{2} \mathbf{b}$ and $\mathbf{3 a}-\mathbf{c}$ led to higher stereoselectivity than the corresponding ether-modified compounds $\mathbf{2 c}$ and $\mathbf{3 d}-\mathbf{f}$. The best results were obtained with 3-O-Ac glucoBox 3a that combines a small 3-O-acyl residue with cyclic 4,6-O-protection, and with bulky ligand Piv glucoBox (2b) without any cyclic protection. These findings are summarised in Scheme 1.

Because of the strong impact of the pyranose position 3 in ligands $\mathbf{3 a}-\mathbf{f}$ on the stereoselectivity, we became interested in elucidating the influence of the stereochemistry at this position by both 3-epimerisation and 3-defunctionalisation. Inversion of the configuration at position 3 to give an allo-configured ligand scaffold, will bring the $3-O$-substituent into a syn-relationship with the oxazoline nitrogen atom and therefore into very close proximity to a coordinated metal centre (Figure 1, I). Deoxygenation of the 3-postion on the other hand will lead to a ligand with comparably little steric shielding of metal centres coordinated by the oxazoline nitrogen atoms (Figure 1, II). As the stereoselectivity of the model reaction for ligands $\mathbf{3 a}-\mathbf{f}$ improved with decreasing steric demand of the 3-O-substituent and since the best results were obtained with acyl-modified ligands, we also set out to prepare a corresponding ligand with a formyl group as the smallest possible acyl residue at the 3-O-position. In this paper we describe the synthesis of new 3-epimerised and 3-deoxygenated carbohydrate bis(oxazolines), the preparation of a 3-O-formate analogue of ligands 3 as well as the testing of these new ligands in stereoselective cyclopropanation.

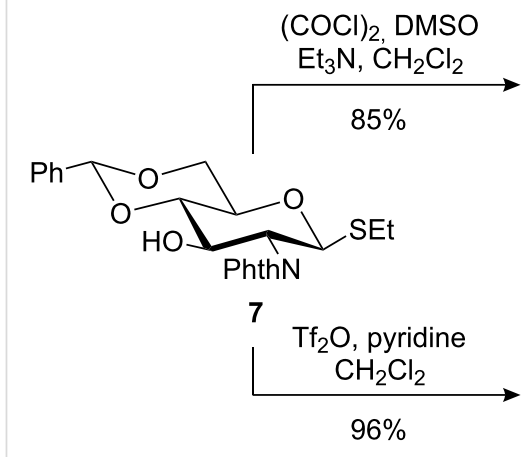

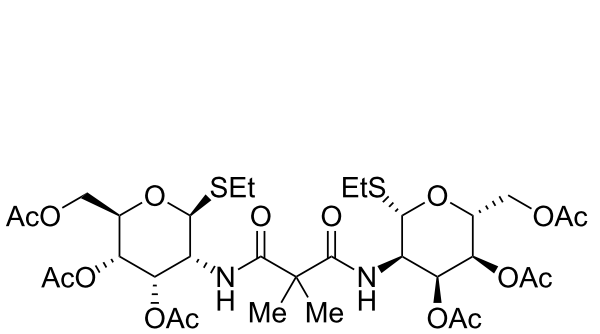

15

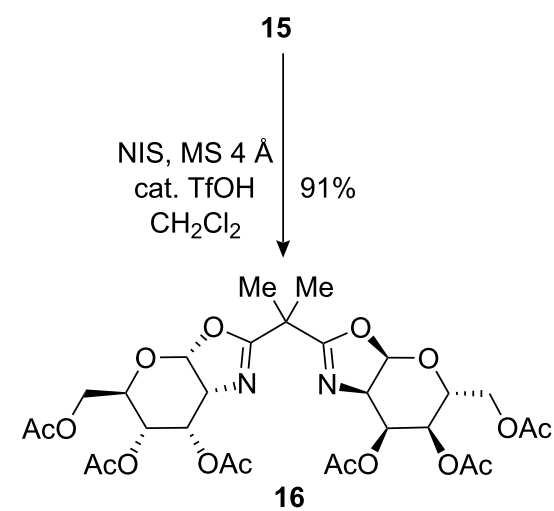

Ac alloBox

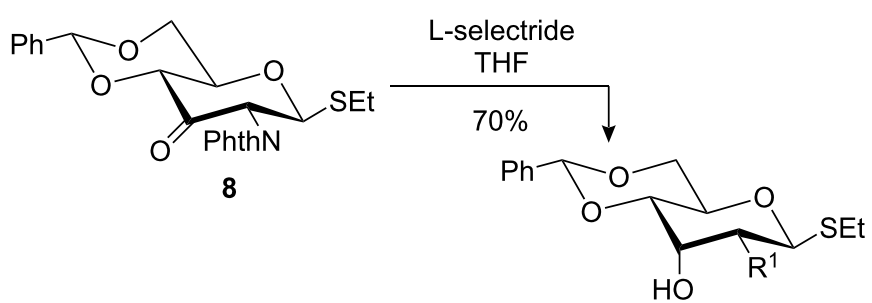

$10 \mathrm{R}^{1}=$ NPhth ethylenediamine $\mathrm{EtOH}$ $90 \%$

1.) $\mathrm{AcOH}(60 \%)$

2.) $\mathrm{Ac}_{2} \mathrm{O}, \mathrm{DMAP}$ pyridine $77 \%$

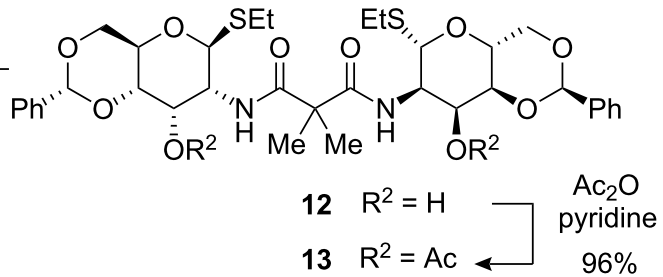

13
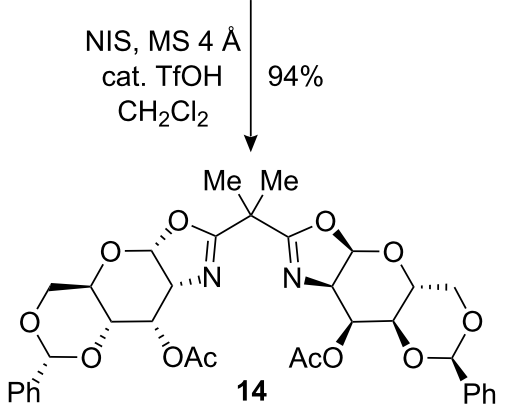

3-O-Ac alloBox 


\section{Results and Discussion}

The synthesis of all new ligands started from the known thioglucoside 7 [24] which was also employed as key intermediate for the preparation of ligands $\mathbf{3 a}-\mathbf{f}[20,21]$ and is accessible from D-glucosamine in 5 steps and $57 \%$ overall yield. To prepare an allo-configured precursor for ligand synthesis, we decided first to use a previously described epimerisation sequence for 7 featuring Swern oxidation and subsequent reduction with sodium borohydride [25]. In our hands this method led to an inseparable product mixture in the second step however, on switching to L-selectride for the stereoselective reduction [26], the allosamine derived thioglycoside $\mathbf{1 0}$ was obtained in good overall yield. For an alternative route, 7 was transformed into the 3-O-triflate 9 and then subjected to nucleophilic displacement with sodium nitrite in the presence of 15 -crown-5 $[27,28]$ to afford $\mathbf{1 0}$ in similar yields as the oxidation-reduction sequence (Scheme 2). After deprotection of the phthalimide (phthN) [29], the free amine 11 was transformed into the 4,6- $O$ benzylidene protected ligand by our standard protocol for the preparation of carbohydrate bis(oxazoline) ligands [20,21] Formation of bis(amide) $\mathbf{1 2}$ with dimethylmalonyl chloride, 3-O-acetylation and subsequent activation of the thioethyl moieties of 13 with NIS [30] for the double cyclisation step, led to benzylidene protected ligand 3-O-Ac alloBox 14 in excellent yield. As noted previously, the presence of a 4,6-O-benzylidene group has a pronounced influence on the conformation adopted by the pyranose scaffold in gluco-configured ligands (Scheme 1, conformers $\mathbf{A}$ and $\mathbf{B}$ ), which in turn has a direct influence on the stereoselectivity in the model reaction. In order to ascertain if a similar conformative effect is also in operation for allo-configured bis(oxazolines), we prepared ligand Ac alloBox 16 with acyclic 4,6-O-protection by the removal of the benzylidene groups from 12 under acidic conditions and per- $O$ acetylation in a one-pot reaction followed by NIS-mediated cyclisation of resulting bis(amide) 15 .

For the preparation of 3-deoxygenated ligands, we planned a defunctionalisation of the key intermediate 7. Surprisingly, a thorough search of the literature revealed only one example of the 3-deoxygenation of a glucosamine-derived thioglycoside, reported by Herdewijn et al. in 2006 [31]. Because the Barton-McCombie deoxygenation [32] failed on their $N$-Troc protected thio aminoglucoside under various conditions, Herdewijn et al. used a sequence via a 3-iodide derivative. To

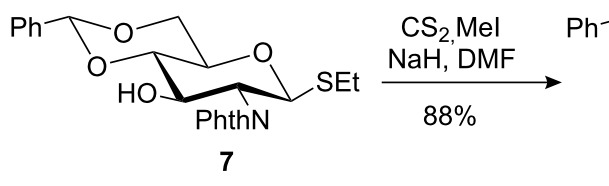<smiles>CC[Si]C1O[C@H](COC(C)=O)[C@@H](OC(C)=O)C[C@H]1NC(=O)C(C)(C)C(=O)N[C@H]1C[C@H](OC(C)=O)[C@H](CC)O[C@H]1CC</smiles>

22

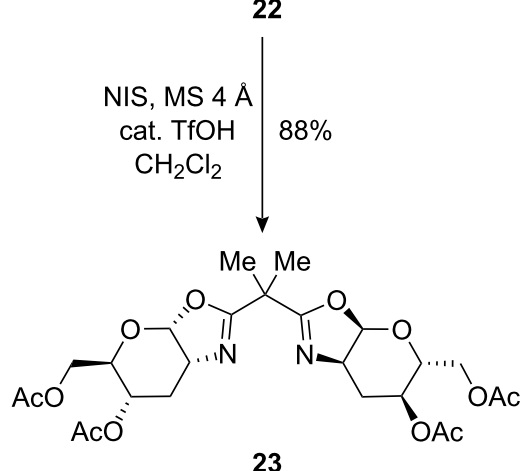

Ac 3-deoxy gluco Box
1.) $\mathrm{AcOH}(60 \%)$

2.) $\mathrm{Ac}_{2} \mathrm{O}, \mathrm{DMAP}$ pyridine $93 \%$<smiles>[Z15][C@@H]1O[C@@H]2COC(c3ccccc3)OC2C[C@@H]1NC(=O)C(C)(C)C(=O)N[C@@H]1C[C@H]2O[C@H](c3ccccc3)OC[C@H]2OC1[Si]CC</smiles>

20

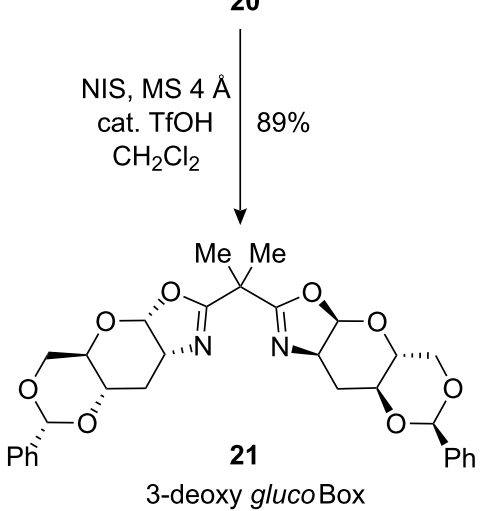

Scheme 3: Preparation of ligands 3-deoxy glucoBox (21) and Ac 3-deoxy glucoBox (23) from key intermediate 7. 
avoid the rather complicated preparation of a 3-iodo derivative, we tried the Barton-McCombie reaction on our phthalimido protected precursor 7 (Scheme 3). Introduction of the 3-xanthogenate with carbon disulfide and methyl iodide yielded $\mathbf{1 7}$, which was cleanly deoxygenated in high yield by tributyltin hydride under standard conditions [32,33]. From the resulting compound 18, the ligands 3-deoxy glucoBox 21 with benzylidene groups and Ac 3-deoxy glucoBox 23 with acyclic 4,6-O-protection were prepared in high overall yields (Scheme 3).

The 3-O-formate analogue of gluco-configured ligands 3 was obtained by treatment of bis(amide) 24 [20,21] with formyl acetate [34] to yield $\mathbf{2 5}$ which was then cyclised to the desired ligand 26 with NIS (Scheme 4).
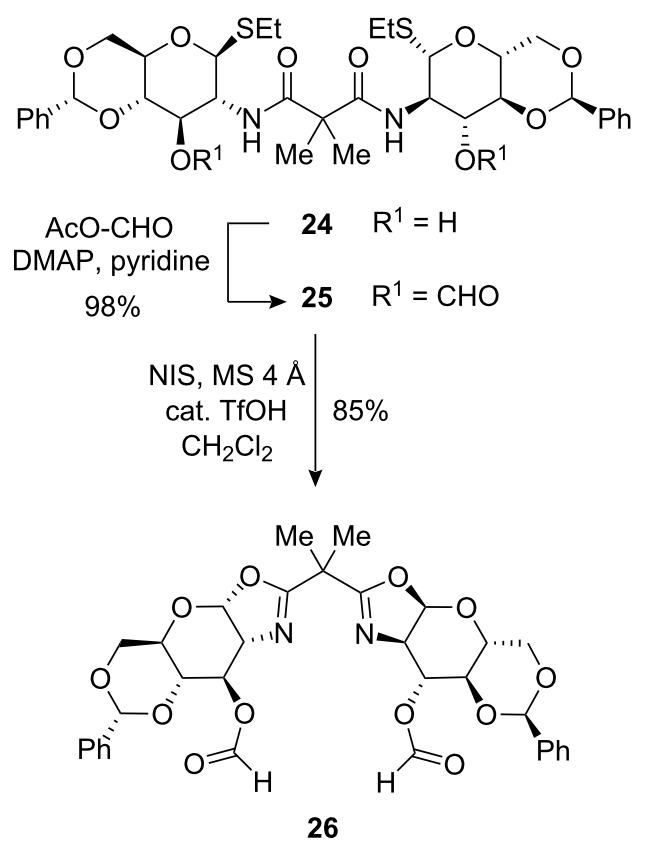

3-O-Formyl glucoBox

Scheme 4: Preparation of ligand 3-O-Formyl glucoBox (26) from bis(amide) 24.

The five new ligands 14, 16, 21, 23 and 26 were now employed in the copper(I) catalysed asymmetric cyclopropanation of styrene (4) with diazoacetate (5) under known conditions $[19,21,22]$ (Table 1). All ligands led to formation of the products trans $\mathbf{6}$ and cis $\mathbf{6}$ in good to excellent yields and the trans/cis ratio was in the typical range (around 70:30) obtained with bis(oxazoline) ligands [22]. However, the enantioselectivities differed dramatically for the new ligands and revealed once again the strong influence of position 3 and the pyranose conformation on the efficiency of the asymmetric induction. The best results were obtained with 3-O-formyl glucoBox 26 which gave trans 6 and cis 6 in $95 \%$ ee and 94\% ee respectively (Table 1 , entry 5).

Figure 2 gives a summary of the results obtained with the new ligands as well as a comparison with the previously reported ligands 2a and 3a. Both, benzylidene-protected ligands 3-O-Ac alloBox 14 and 3-deoxy glucoBox 21 gave only racemic products while their counterparts Ac alloBox $\mathbf{1 6}$ and Ac 3-Deoxy glucoBox 23 lacking cyclic 4,6-O-protection led to substantial asymmetric induction. This demonstrates that the dramatic conformational effect of the pyranose scaffold on stereoselectivity, which was first observed for gluco-configured ligands $\mathbf{2}$ and $\mathbf{3}$, is also in operation in allo- and 3-deoxy glucoligands. However, while benzylidene protection in 3-O-Ac glucoBox 3a led to improved asymmetric induction in comparison to ligand Ac glucoBox 2a lacking cyclic protection, the opposite was observed for the allo- and 3-deoxy-ligands. The strong influence of the configuration of pyranose position 3 on stereoselectivity becomes apparent by a comparison of ligand 3-O-Ac glucoBox 3a to its 3-epimerised and 3-defunctionalised counterparts 14 and 21: Both modifications, inversion of the configuration in allo-ligand $\mathbf{1 4}$ and 3-defunctionalisation in $\mathbf{2 1}$ result in a complete loss of stereoselectivity in the model reaction, whilst 3-O-Ac glucoBox 3a provides the products in $93 \%$ ee and $82 \%$ ee respectively. Finally, gluco-configured ligand 26 with a 3-O-formyl residue led to higher stereoselectivities (95\% ee and $94 \%$ ee for trans 6 and cis 6 respectively) than 3-O-acetylated ligand 3a. This confirms the trend we initially observed for gluco-configured ligands. A decrease in steric bulk of 3-O-acyl substituents results in improved asymmetric induction of the ligand in the cyclopropanation reaction: ee for 3-O-Piv 3c $<3$ - $O$-Bz 3b $<3$-O-Ac 3a $<3$-O-Formyl 26. Thus, of all carbohydrate-derived bis(oxazolines) prepared by us, ligand $\mathbf{2 6}$ led to the best enantioselectivities for cyclopropanes trans $\mathbf{6}$ and cis $\mathbf{6}$.

\section{Conclusion}

We have prepared new derivatives of gluco-configured bis(oxazoline) ligands $\mathbf{2}$ and $\mathbf{3}$ with 3-epimerisation or 3-defunctionalisation in the pyranose scaffold. Application in stereoselective cyclopropanation as a model reaction highlighted the strong impact of modifications at the pyranose position 3 on the asymmetric induction exerted by carbohydrate-based ligands. Furthermore, the previously observed conformational effect of cyclic 4,6-O-benzylidene protection on stereoselectivity is also in operation in the new derivatives. Introduction of a 3-Oformate in glucoBox ligands led to improved stereoselectivities compared to the corresponding 3-O-acetate. This underlines our previous findings that the best results for gluco-configured ligands are obtained with small acyl-based 3-O-substitutents. 
Table 1: Cyclopropanations with allo-configured ligands 14 and 16, 3-deoxygenated ligands 21 and 23 and 3-O-formylated, gluco-configured ligand 26.

\begin{tabular}{|c|c|c|c|c|c|}
\hline \multirow[b]{2}{*}{ Entry } & $\widehat{N}_{4}^{+} \mathrm{N}_{5} \mathrm{CO}_{2} \mathrm{E}$ & $\begin{array}{l}\text { Ligand } \\
\text { CuOTf }\end{array}$ & $\underset{\mathrm{h}}{\stackrel{(1 \mathrm{~mol} \%)}{\longrightarrow}}$ & $\mathrm{O}_{2} \mathrm{Et}+{ }_{\mathrm{Ph}}^{(R) /}$ & \\
\hline & Ligand & Yield [\%] ${ }^{a}$ & trans/cis ${ }^{\mathrm{b}}$ & ee trans $[\%]^{b}$ & ee $\operatorname{cis}[\%]^{\mathrm{b}}$ \\
\hline 1 & 3-O-Ac alloBox (14) & 75 & $66: 34$ & rac. & rac. \\
\hline 2 & Ac alloBox (16) & 79 & $70: 30$ & 71 & 87 \\
\hline 3 & $\begin{array}{l}\text { 3-deoxy glucoBox } \\
\text { (21) }\end{array}$ & 86 & $69: 31$ & rac. & rac. \\
\hline 4 & $\begin{array}{l}\text { Ac 3-deoxy } \\
\text { glucoBox (23) }\end{array}$ & 75 & $74: 26$ & 78 & 72 \\
\hline 5 & $\begin{array}{l}\text { 3-O-formyl } \\
\text { glucoBox (26) }\end{array}$ & 95 & $71: 29$ & 95 & 94 \\
\hline
\end{tabular}

alsolated yield after chromatography.

betermined by GC on a chiral stationary phase.

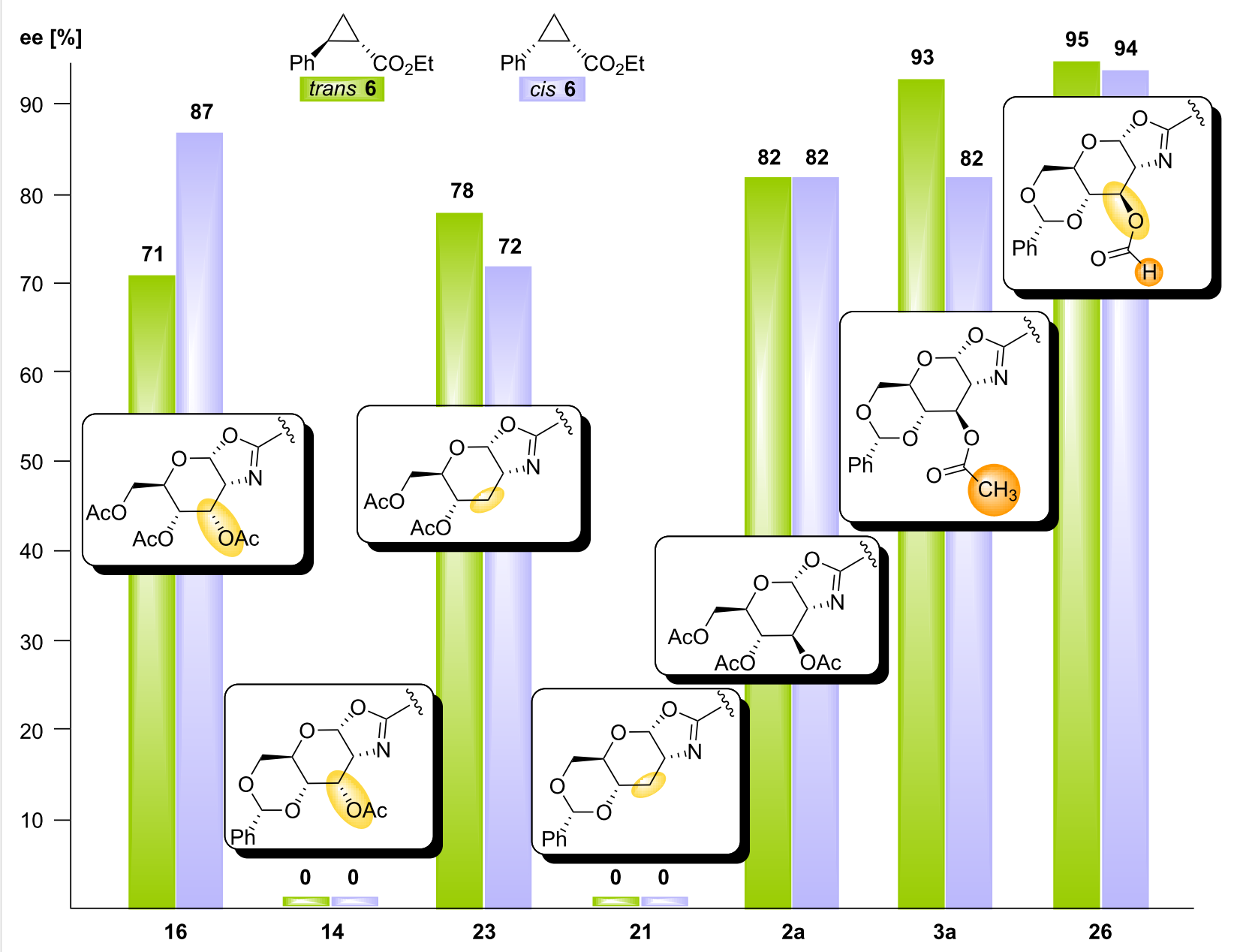

Figure 2: Impact of structural ligand modifications on the stereoselectivity of cyclopropanations. 
The observed steric, configurational and conformational effects are as yet not fully understood and investigations to elucidate their origins are currently under way.

\section{Supporting Information}

Supporting information contains full experimental details for the preparation of all new ligands and general conditions for cyclopropanations using glucoBox ligands and copper(I) triflate.

\section{Supporting Information File 1}

Experimental details.

[http://www.beilstein-journals.org/bjoc/content/ supplementary/1860-5397-6-23-S1.pdf]

\section{Acknowledgements}

We thank the German Research Foundation (DFG) and the Volkswagen Foundation for generous financial support.

\section{References}

1. Cullen, W. R.; Sugi, Y. Tetrahedron Lett. 1978, 19, 1635-1636. doi:10.1016/S0040-4039(01)94626-X

2. Jackson, R.; Thompson, D. J. J. Organomet. Chem. 1978, 159, C29-C31. doi:10.1016/S0022-328X(00)92235-6

3. Selke, R. React. Kinet. Catal. Lett. 1979, 10, 135-138. doi:10.1007/BF02075980

4. Sinou, D.; Descotes, G. React. Kinet. Catal. Lett. 1980, 14, 463-466. doi:10.1007/BF02061353

5. Hale, K. J. Monosaccharides: Use in synthesis as chiral templates. In Second Supplement to the Second Edition of Rodd's Chemistry of Carbon Compounds; Sainsbury, M., Ed.; Elsevier: Amsterdam, The Netherlands, 1993; Vol. 1E/F/G, pp 273-313.

6. Steinborn, D.; Junicke, H. Chem. Rev. 2000, 100, 4283-4318. doi:10.1021/cr9903050

7. Diéguez, M.; Pàmies, O.; Claver, C. Chem. Rev. 2004, 104, 3189-3215. doi:10.1021/cr0306889

8. Diéguez, M.; Pàmies, O.; Ruiz, A.; Díaz, Y.; Castillón, S.; Claver, C. Coord. Chem. Rev. 2004, 248, 2165-2192. doi:10.1016/j.ccr.2004.04.009

9. Díaz, Y.; Castillón, S.; Claver, C. Chem. Soc. Rev. 2005, 34, 702-713. doi:10.1039/b400361f

10. Diéguez, M.; Claver, C.; Pàmies, O. Eur. J. Org. Chem. 2007, 4621-4634. doi:10.1002/ejoc.200700082

11. Boysen, M. M. K. Chem.-Eur. J. 2007, 13, 8648-8659. doi:10.1002/chem.200701010

12. Benessere, V.; De Roma, A.; Del Litto, R.; Ruffo, F. Coord. Chem. Rev. 2010, 254, 390-401. doi:10.1016/j.ccr.2009.05.001

13. Desimoni, G.; Faita, G.; Jørgensen, K. A. Chem. Rev. 2006, 106, 3561-3651. doi:10.1021/cr0505324

14. McManus, H. A.; Guiry, P. J. Chem. Rev. 2004, 104, 4151-4202. doi:10.1021/cr040642v

15. Gläser, B.; Kunz, H. Synlett 1998, 53-54. doi:10.1055/s-1998-3124

16. Yonehara, K.; Hashizume, T.; Mori, K.; Ohe, K.; Uemura, S. Chem. Commun. 1999, 415-416. doi:10.1039/a810041a
17. Mata, Y.; Diéguez, M.; Pàmies, O.; Claver, C. Org. Lett. 2005, 7, 5597-5599. doi:10.1021/ol052176h

18. Hartinger, C. G.; Nazarov, A. A.; Galanski, M.; Reithofer, M.; Keppler, B. K. J. Organomet. Chem. 2005, 690, 3301-3308. doi:10.1016/j.jorganchem.2005.03.062

19. Irmak, M.; Groschner, A.; Boysen, M. M. K. Chem. Commun. 2007, 177-179. doi:10.1039/b612986b

20. Minuth, T.; Boysen, M. M. K. Synlett 2008, 1483-1486. doi:10.1055/s-2008-1078419

21. Minuth, T.; Irmak, M.; Groschner, A.; Lehnert, T.; Boysen, M. M. K. Eur. J. Org. Chem. 2009, 997-1008. doi:10.1002/ejoc.200801035

22. Evans, D. A.; Woerpel, K. A.; Hinman, M. M.; Faul, M. M. J. Am. Chem. Soc. 1991, 113, 726-728. doi:10.1021/ja00002a080

23. Loewenthal, R. E.; Abiko, A.; Masamune, S. Tetrahedron Lett. 1990, 31, 6005-6008. doi:10.1016/S0040-4039(00)98014-6

24. Lönn, H. Carbohydr. Res. 1985, 139, 105-113. doi:10.1016/0008-6215(85)90011-4

25. Kajihara, Y.; Kodama, H.; Endo, T.; Hashimoto, H. Carbohydr. Res. 1998, 306, 361-378. doi:10.1016/S0008-6215(97)10093-3

26. Emmerson, D. P. G.; Villard, R.; Muganini, C.; Batsanov, A.; Howard, J. A. K.; Hems, W. P.; Tooze, R. P.; Davis, B. G. Org. Biomol. Chem. 2003, 1, 3826-3838. doi:10.1039/b309715n

27. Pei, Z.; Dong, H.; Caraballo, R.; Ramström, O. Eur. J. Org. Chem. 2007, 4927-4934. doi:10.1002/ejoc.200700364

28. Luo, S.-Y.; Kulkarni, S. S.; Chou, C.-H.; Liao, W.-M.; Hung, S.-C. J. Org. Chem. 2006, 71, 1226-1229. doi:10.1021/jo051518u

29. Huang, L.; Wang, Z.; Li, X.; Ye, X.; Huang, X. Carbohydr. Res. 2006, 341, 1669-1679. doi:10.1016/j.carres.2006.01.007

30. Sherman, A. A.; Yudina, O. N.; Mironov, Y. V.; Sukhova, E. V.; Shashkov, A. S.; Menshov, V. M.; Nifantiev, N. E. Carbohydr. Res. 2001, 336, 13-46. doi:10.1016/S0008-6215(01)00213-0

31. Sun, D.-Q.; Busson, R.; Herdewijn, P. Eur. J. Org. Chem. 2006, 5158-5166. doi:10.1002/ejoc.200600515

32. Barton, D. H. R.; McCombie, S. W. J. Chem. Soc., Perkin Trans. 1 1975, 1574-1585. doi:10.1039/P19750001574

33. Gemma, E. Synthesis of Oligosaccharides for Interaction Studies with Various Lectins. Ph.D. Thesis, University of Stockholm, Sweden, 2005

34. Elders, N.; Schmitz, R. F.; de Kanter, F. J. J.; Ruijter, E.; Groen, M. B.; Orru, R. V. A. J. Org. Chem. 2007, 72, 6135-6142. doi:10.1021/jo070840x

\section{License and Terms}

This is an Open Access article under the terms of the Creative Commons Attribution License (http://creativecommons.org/licenses/by/2.0), which permits unrestricted use, distribution, and reproduction in any medium, provided the original work is properly cited.

The license is subject to the Beilstein Journal of Organic Chemistry terms and conditions: (http://www.beilstein-journals.org/bjoc)

The definitive version of this article is the electronic one which can be found at: doi:10.3762/bjoc. 6.23 